\title{
A 2-D Mathematical Model of Vortex Induced Vibration Driven Bladeless Wind Turbine
}

\author{
Sirada Saengsaen ${ }^{1,}$, Chawin Chantharasenawong ${ }^{2}$ and Tsung-Liang $\mathrm{Wu}^{3}$ \\ ${ }^{1}$ The Joint Graduate School of Energy and Environment, King Mongkut's University of Technology Thonburi, Bangkok, Thailand \\ ${ }^{2}$ Department of Mechanical Engineering, Faculty of Engineering, King Mongkut's University of Technology Thonburi, Bangkok, Thailand \\ ${ }^{3}$ Department of Mechanical and Automation Engineering, College of Engineering, National Kaohsiung University of Science and Technology, \\ Kaohsiung, Taiwan
}

\begin{abstract}
Bladeless wind turbine (BWT) is a flexible cylindrical structure that extracts energy from wind by utilising vortex-induced vibration (VIV) - aerodynamic forces and the resulting structural vibration. This work focuses on taking possible advantage of the increase in lift forces in the similar fashion to birds flying in a V-formation. The purposes of the present study are 1) to study the flow pattern and characteristic around two BWTs which are cylindrical structures in the same flow field and 2) to study the extra lift force generation of the system. 2-D CFD models are used to simulate flow of stationary cylinder of BWTs at $\mathrm{Re}=10^{5}$. The two different turbulent models, Reynolds Averaged Navier-Stokes shear-stress transport k $\omega$ (RANS-SST k $\omega$ ) and Detached Eddy Simulation shearstress transport $\mathrm{k} \omega$ (DES-SST $\mathrm{k} \omega$ ) are investigated. The results show that only DES-SST k $\omega$ gives converged results, therefore, DES-SST k $\omega$ is selected for the additional studies of two cylindrical structures. From 2-D CFD simulation, the one BWT in flow field case produces lift coefficient 0.851. In the case of two BTWs in same flow field (BWT no. 2 is located at $x=3 \mathrm{D}, \mathrm{y}=0 \mathrm{D}$, directly downstream of two cylinders), BTW no. 1 and BWT no. 2 show greater lift coefficients of 0.893 and 1.841 , respectively. This result indicates that the kinetic energy generation of the downstream BWT in the two BWTs system in this study is greater than the baseline BWT with an increase of $116 \%$ of lift. Further work is needed to determine the optimum location of the behind wind turbine for greater lift and result to increasing of energy produce of the system.
\end{abstract}

\section{Introduction}

Recently, a new concept of wind energy harvesting called bladeless wind turbine (BWT) has been developed, which is based on vortex-induced vibration [1] as shown in figure 1. VIV is a phenomenon that occurs when shedding vortices (a von Karman vortex street) exert oscillatory lift forces on a body perpendicular to flow direction [2-3] as shown in figure 2. These vortices cause the BWT to oscillate in traverse flow. The energy harvesting of these oscillations can occur from interactions between the elastic structure and fluid forces, which surrounding fluid transfer energy from oscillation to the structure [4]. When the vortex shedding frequency is close to the structural natural frequency, subsequently the structure oscillates in resonance called lock-in. The main flow in the lock-in region provides energy to excite the structural vibrations, while the flow in the non-lock-in region damps the structural vibrations [5]. When there are multi BWTs, an example which demonstrates the effect of vortex shedding from multi bluff body is the birds flying in a V-formation. As a bird flaps its wings, a rotating vortex of air rolls off each of its wingtips. The vortices behind bird are continuously pushed downwards (downwash), and the air behind to the sides gets pushed

\footnotetext{
a Corresponding author: sirada.s@mail.kmutt.ac.th
}

upwards (upwash). If another bird flies in either of these upwash zones, it gets additional lift then it can save energy by seeking the air flow created by its flock-mate. The BWTs driven by VIV behave likewise a flock of bird, therefore, the layout design and performance analysis of VIV driven BWT should be investigated with parameters

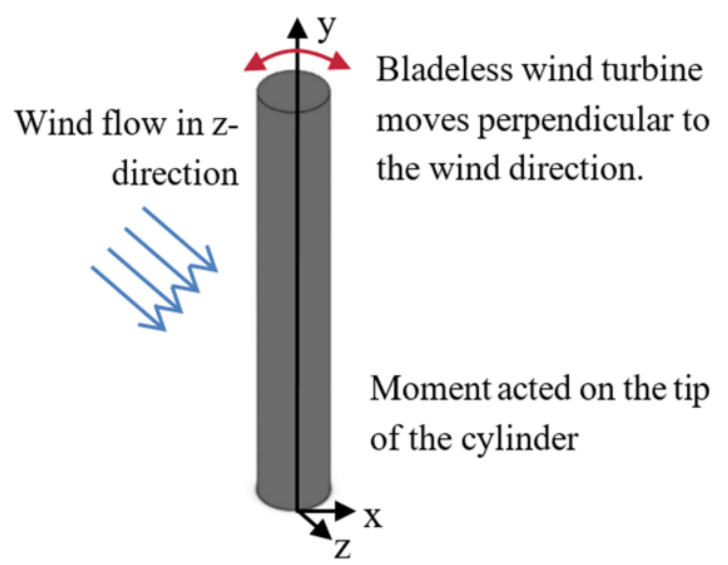

Figure 1. The schematic model of a circular rigid cylinder mounted on a flexible BWT [3]. 
in lock-in phenomena to maximize the extraction of power from wind energy.

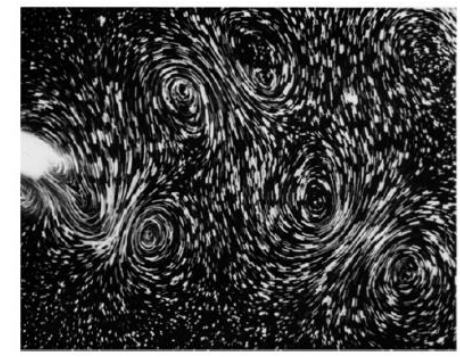

Figure 2. Visualization of the Von Kármán vortex downstream of a cylinder in air flow. Cylinder is towed through fluid in a sinusoidal trajectory to the left [4].

Model of VIV driven BWT requires fluid flow forces and structural force-vibration model which obtained the Navier-Stokes equations. The analytical solution of the Navier-Stokes equations are very difficult to solve. In addition, the three-dimensional (3-D) computational simulation is also complex and difficult due to nonsteady of vortices shedding of VIV bladeless wind turbines. Alternatively, a two-dimensional (2-D) flow model can solve the problem by consider a transverse flow induced force on each slice of the structure [6-7] to find the lift force. The 2-D simulation of two cylinders at $\mathrm{Re}=100$ [8] and 3-D simulation of three cylinders at high $\mathrm{Re}$ [9] were concluded that VIV influence on lift generation by effect of spacing between cylinders. The simulation of [9] used LES and its solution is reliable. However, LES has high cost computational as well as work investigated only one case of angle between downstream and upstream cylinders. On the other hand, a $2-\mathrm{D}$ case for $\mathrm{Re}=10^{4}$ with the Reynolds Averaged Navier-Stokes (RANS) shear stress transport k- $\omega$ (SST k$\omega)$ model, which involves minimal computational cost, is reliable and appropriate for analysing the characteristics of VIV in [7]. The results show good agreement of maximum lift force amplitude by compared with detached eddy simulation (DES) and direct numerical simulation and LES models. In addition, M.C. Ong et al. [10] show 2D-URANS simulation of flow past a cylinder at $\mathrm{Re}$ in the supercritical and upper-transition flow regimes, i.e. $\operatorname{Re}>10^{6}$ that give satisfy agreements with the published experimental data. On the other hand, Stringer [11] found that of Reynolds number greater than $10^{5}$, RANS k- $\omega$ model gives drag coefficient with variable high value from experiment and Rosetti et al. [12] simulate flow past a cylinder using 2-D RANS-SST $\mathrm{k} \omega$ at $\mathrm{Re}=10^{5}$ and $C_{D}$ and $C_{L}$ result is error within $30 \%$. However, RANS model is benefit in computational cost even its results has hesitation in high Reynolds number.

The purposes of present study are 1) to study the flow pattern and characteristic around two BWTs which are cylindrical structures in the same flow field and 2) to quantify the impact of lift force generation of the system at $\mathrm{Re}=10^{5}$. The present study considers the 2-D CFD model as flow over stationary cylinder diameter $\mathrm{D}$ of BWTs. The numerical method is finite volume method (FVM) which in ANSYS Fluent to solve problems. Turbulent properties of the flow are primarily solved using RANS and DES and compare results to choose the suitable model. Then, simulation results of one BWT and Two BWTs are comparison of generated lift.

\section{Determination of lift and drag forces}

The flow around the cylinder causes varying fluid pressure and shear stress, and the resulting fluid force acting on the cylinder can be found by integrating the contributions around the circumference. The force component in the direction of the flow is called drag. The force component normal to the flow is called lift. The forces are commonly expressed as dimensionless drag (1) and lift coefficients (2), defined as

$$
\begin{aligned}
C_{D} & =\frac{F_{D}}{\frac{1}{2} \rho D U^{2}} \\
C_{L} & =\frac{F_{L}}{\frac{1}{2} \rho D U^{2}}
\end{aligned}
$$

where, $U$ is free steam velocity, $C_{D}$ is the drag coefficient, $\mathrm{F}_{\mathrm{D}}$ drag force per unit length and $\rho$ is the fluid density. While $C_{L}$ is the lift coefficient and $F_{L}$ lift force per unit length. Lift force can be formulated as

$$
F_{L}(t)=\frac{1}{2} \rho D U^{2} C_{L} \sin \left(\omega_{s} t\right)
$$

where, $\omega_{s}$ than frequency of vortex shedding.

The recorded lift data will give the sinusoidal term which can be calculated to root-mean-square due to that athematic mean is equal to zero which is useless for analyse data. The root-mean-square value of fluctuating lift coefficient $\left(\mathrm{C}_{\text {Lrms }}\right)$ is given by

$$
C_{L r m s}=\frac{\sqrt{\frac{1}{n}\left(F_{L 1}^{2}+F_{L 2}^{2}+F_{L 3}^{2} \ldots+F_{L n}^{2}\right)}}{\frac{1}{2} \rho D U^{2}}
$$

\section{Simulation modelling}

\subsection{Properties of fluid}

The fluid properties in this simulation are as follows, $\mathrm{Re}=10^{5}$ diameter; $D=0.30 \mathrm{~m}$ and free stream velocity; $U=5.228 \mathrm{~m} / \mathrm{s}$ which is compatible with the experiment case in the further work, and dynamic viscosity; $\mu=1.84610^{-5} \mathrm{~kg} / \mathrm{m}-\mathrm{s}$ and air density; $\rho=1.177 \mathrm{~kg} / \mathrm{m}-\mathrm{s}$ from temperature of ambient air of $300 \mathrm{~K}$.

\subsection{Model Validation}

The one BWT case (one cylinder) is used as a baseline case of lift generation. In order to find the reliable result, grid independency is first established. The Turbulent Models for solving one BWT simulation are RANS-SST

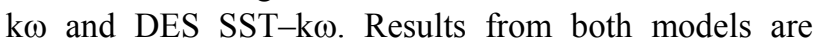
compared afterward. The computational domain in this study is shown in figure 3 and is referenced from [9]. The example of mesh is shown in figure 4 . The time step size 
$(\Delta t)$ is one of the significant parameters which affect the results. The time step size has been studied in $[7,10]$ and $\Delta \mathrm{t}=0.001$ and $\Delta \mathrm{t}=0.005 \mathrm{~s}$ have been suggested. This present work also studies time step size independence then all simulations are used time step $0.005 \mathrm{~s}$ and total maximum iteration is $200,000 \mathrm{~s}$ and all residual target is $10^{-6}$. This study uses $C_{D}$ and $C_{\mathrm{L}, \mathrm{rms}}$ to indicate the convergence values. The correct solution does not directly rely on the converged solution and the quality of the grid is important for the accuracy and reliability of the solution. The simulation results of different grid size at $\operatorname{Re}=10^{5}$ are given in figure $5-6$. The $C_{D}$ is between 0.9 - 1.2 and $\mathrm{C}_{\mathrm{L}, \mathrm{rms}}$ is between $0.45-0.6$ [11-12] using to reference for simulation validation and difference. In figure 5 and figure 6 show convergence of the $C_{D}$ and $C_{L}$ values. The $C_{D}$ is corresponding to reference within range of 0.9 to 1.2 . The $C_{L}$ is over-predicted values with 15.33 $-41.83 \%$ difference which is probably due to the overprediction of pressure. It can be seen that RANS-SST k $\omega$ model show fluctuation in both drag and lift coefficient meanwhile DES - SST k $\omega$ give the convergent results. Therefore, DES-SST k $\omega$ is selected turbulent model to conduct for all the 2-D simulations cases in present study. The simulation number 2 is selected due to low computation cost.

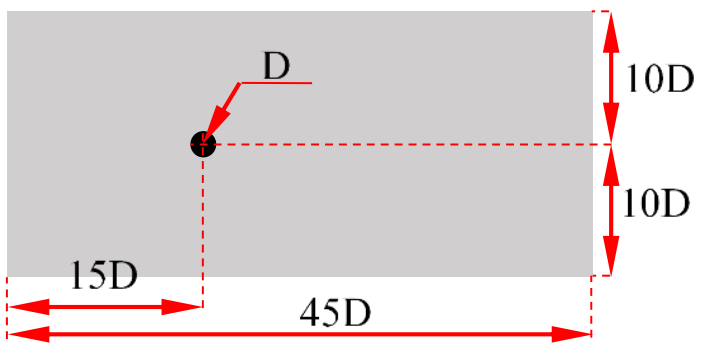

Figure 3. A computational domain $(20 \mathrm{D} \times 45 \mathrm{D})$ for one BWT.

a)

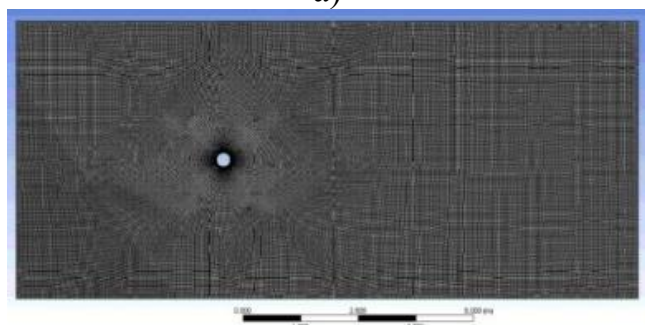

b)

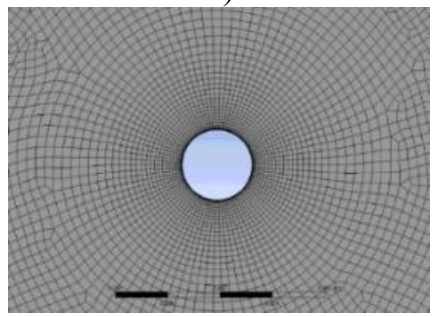

Figure 4. The computational mesh $\left(\operatorname{Re}=10^{5}\right)$; a) far field meshes, b) detail of Quadrilateral mesh of one BWT. The number of mesh is 54,228 elements.

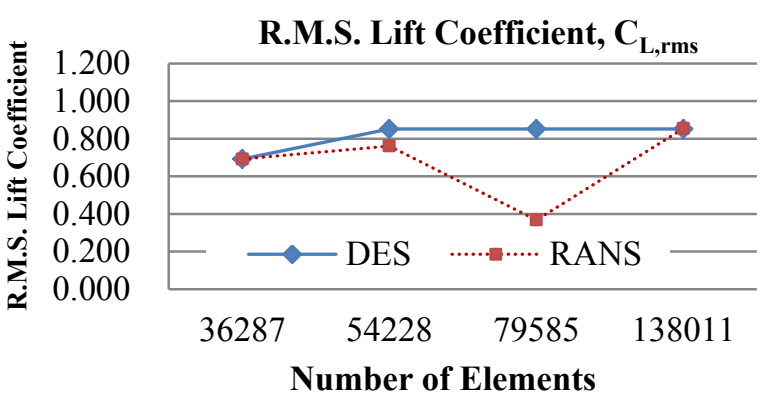

Figure 6. The convergence of the $\mathrm{C}_{\mathrm{L}, \mathrm{rms}}$ values.

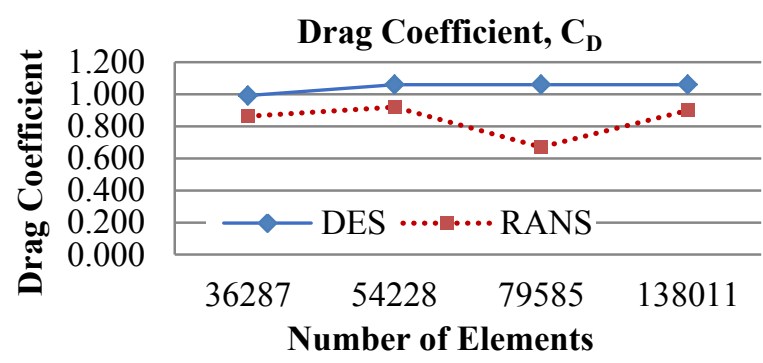

Figure 5. The convergence of the $C_{D}$ values.

\section{Two Bladeless Wind Turbine (Two Cylinders)}

The distance between center-to-center of two cylinders is greater than 1.4 diameters, the vertex shedding will occur [3]. To initiate the distance between two cylinders, the primary simulation is set $\mathrm{x}=3$ times of diameter and $\mathrm{y}=$ 0 . The size of computational domain is same as simulation of one cylinder (seeing figure 7). The time step size $(\Delta \mathrm{t})$ for all simulation are $0.005 \mathrm{~s}$ and total maximum iteration is 200,000 and all residual target is $10^{-6}$. The grid are 56,449 elements for simulation of two cylindrical BWT in the same flow field, $C_{D}$ and $C_{L}$ results at steady state are shown in figure 8 to figure 10 and frequency and Strouhal number of upstream and downstream are $\mathrm{f}_{\mathrm{s} 1}=4.26$, and $\mathrm{St}_{1}=0.245$, and $\mathrm{f}_{\mathrm{s} 2}=4.23$, and $\mathrm{St}_{2}=0.243$, respectively.

a)

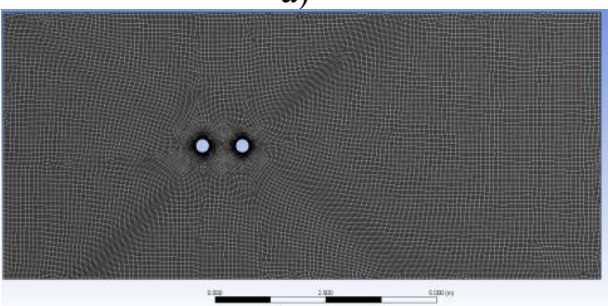

b)

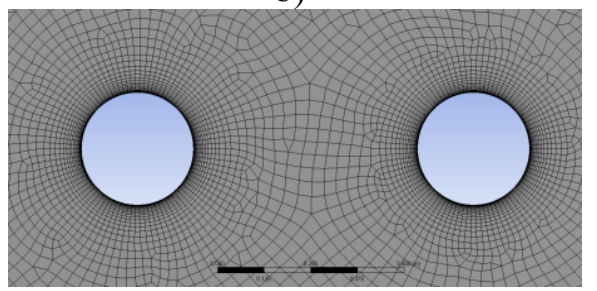

Figure 7. The computational mesh $\left(\operatorname{Re}=10^{5}\right)$; a) far field meshes, b) detail of Quadrilateral mesh of two cylindrical BWTs simulation. The number of mesh is 56,449 elements. 
Figure 10 also shows that having two cylindrical BWTs in the flow field significantly affects lift and drag coefficients. This is clearly illustrated by the larger loop in the graph. In the baseline one BWT case, the rootmean-square lift coefficient is 0.851 while in the case of two cylinders the upstream and downstream cylinders exhibit root-mean-square values of lift coefficient of 0.893 and 1.841 , respectively. A $116.2 \%$ increase in magnitude of lift coefficient root-mean-square value is observed in the downstream cylinder. This is due to the induced circulation contributed by the shed vortices of the upstream cylinder on the downstream cylinder as illustrated in figure 11, which consequently affects the pressure distribution patterns around the downstream cylinder. Meanwhile, drag force of both 2 BWTs are reduced comparing of having one BWT in flow filed, accordingly the pressure backward of frontal BWT. The comparison of one BTW in flow filed and two BWTs in same flow field show in Table 1.

Table 1. The comparison of simulation results of one and two cylindrical BWTs $(x=3 \mathrm{D}, \mathrm{y}=0 \mathrm{D})$.

\begin{tabular}{|l|c|c|c|c|c|}
\hline \multirow{2}{*}{ RESULTS } & \multirow{2}{*}{$\begin{array}{c}\text { One } \\
\text { BWT }\end{array}$} & \multicolumn{4}{|c|}{ Two BWTs } \\
\cline { 3 - 6 } & & No. 1 & difference & No. 2 & difference \\
\hline Drag Force [N] & 5.115 & 4.238 & $-17.15 \%$ & 3.404 & $-33.45 \%$ \\
\hline Lift Force [N] & 4.109 & 4.308 & $4.84 \%$ & 8.885 & $116.23 \%$ \\
\hline $\mathrm{C}_{\mathrm{D}}$ & 1.060 & 0.878 & $-17.17 \%$ & 0.705 & $-33.49 \%$ \\
\hline $\mathrm{C}_{\mathrm{L}, \mathrm{rms}}$ & 0.851 & 0.893 & $4.94 \%$ & 1.841 & $116.33 \%$ \\
\hline $\begin{array}{l}\text { Vortex Shedding } \\
\text { Frequency (f } \\
{[\mathrm{Hz})}\end{array}$ & 4.72 & 4.26 & $-9.75 \%$ & 4.23 & $-10.38 \%$ \\
\hline $\begin{array}{l}\text { Strouhal Number } \\
\text { (St) }\end{array}$ & 0.271 & 0.245 & $-9.59 \%$ & 0.243 & $-10.33 \%$ \\
\hline
\end{tabular}

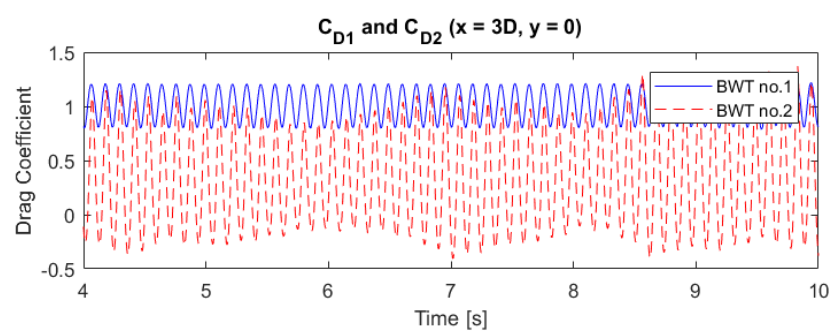

Figure 8. Drag Coefficient of BWT no.1 and BWT no.2

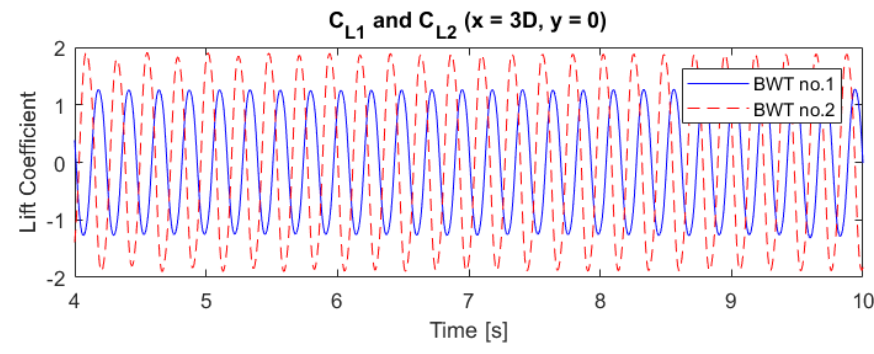

Figure 9. Lift coefficient of BWT no.1 and BWT no.2.

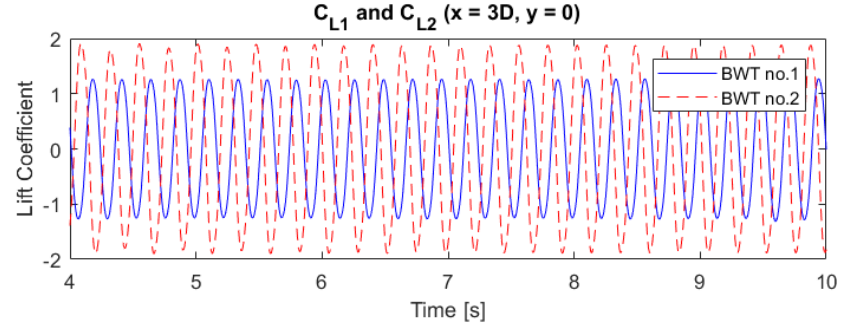

Figure 10. Comparison of $\mathrm{C}_{\mathrm{D}}$ and $\mathrm{C}_{\mathrm{L}, \mathrm{rms}}$ of one BWT and two BWTs at steady state.

\section{Conclusion}

RANS-SST $k \omega$ model gives fluctuating results of CD and $\mathrm{C}_{\mathrm{L}, \mathrm{rms}}$ meanwhile DES-SST $\mathrm{k} \omega$ gives the convergent simulation results in flow over stationary cylinder at $\mathrm{Re}=$ $10^{5}$. The DES model is applied to simulate the system of two BWTs in the same flow field. The BWT no.1 affects BWT no.2 with up to $116.33 \%$ increase of magnitude of lift which means that vibration increases, and the energy produce of the system are increased. For the further work, the research is needed to determine the optimum location of the downstream wind turbine for greater lift increase and result in maximum of energy production of the system r. Moreover, it should be performed the lift force analysis at the difference Reynold number as well as conducted an experiment in wind tunnel to validate the potentiality of BWT.

\section{References}

1. D.J.Y. Villarreal, VIV resonant wind generators, www.vortextbladeless.com, June 7th (2018)

2. C.H. Williamson and R. Govardhan, J WIND ENG IND AEROD 96, 6, 713-735 (2008)

3. R.D. Blevins, Flow-Induced Vibrations (Nostrand Reinhold, 1990)

4. A.B. Rostami and M. Armandei, RENEW SUST ENERG REV 70, 193-214 (2017)

5. R. Bourguet, G.E. Karniadakis and M.S. Triantafyllou, J FLUID STRUCT 27, 838-847 (2011)

6. R.H.J. Willden and J.M.R. Graham, J FLUID STRUCT 19, 123-140 (2001)

7. N.B. Khan, Z. Ibrahim, L.T.T. Nguyen, M.F. Javed and M. Jameel, PLoS One 12,10 (2017)

8. A. Mittal and V. Kumar, J FLUID STRUCT 15, 5, 717-736 (2001)

9. H. Sayyaadi and A. Motekallem, IJCOE 4, 1-10 (2016)

10. M.C. Ong, T. Utnes, L.E. Holmedal, D. Myrhaug, and B. Pettersen, MAR STRUCT 22, 142-153 (2009)

11. R.M. Stringer, J. Zang and A.J. Hillis, Ocean Eng. 87, 1-9 (2014)

12. G.F. Roseri, G. Vaz and A.L.C. Fujarra, OMAE2012 (to be published) 


\section{Reference List}

\begin{tabular}{|c|c|c|c|c|c|c|}
\hline Reference & [1] & [2] & [3] & [4] & [5] & [6] \\
\hline Authors & $\begin{array}{c}\text { D.J.Y. } \\
\text { Villarreal }\end{array}$ & $\begin{array}{c}\text { C.H. } \\
\text { Williamson, } \\
\text { R. Govardhan }\end{array}$ & $\begin{array}{l}\text { R.D. } \\
\text { Blevins, }\end{array}$ & $\begin{array}{l}\text { A.B. Rostami, } \\
\text { M. Armandei }\end{array}$ & $\begin{array}{c}\text { R. Bourguet, } \\
\text { G.E. } \\
\text { Karniadakis and } \\
\text { M.S. } \\
\text { Triantafyllou }\end{array}$ & $\begin{array}{l}\text { R.H.J. } \\
\text { Willden and } \\
\text { J.M.R. } \\
\text { Graham }\end{array}$ \\
\hline Journal title & & $\begin{array}{l}\text { J WIND ENG } \\
\text { IND AEROD }\end{array}$ & & $\begin{array}{c}\text { RENEW SUST } \\
\text { ENERG REV }\end{array}$ & $\begin{array}{l}\text { J FLUID } \\
\text { STRUCT }\end{array}$ & $\begin{array}{l}\text { J FLUID } \\
\text { STRUCT }\end{array}$ \\
\hline $\begin{array}{c}\text { Book title, } \\
\text { Proceedings title }\end{array}$ & $\begin{array}{l}\text { VIV resonant } \\
\text { wind } \\
\text { generators }\end{array}$ & & $\begin{array}{c}\text { Flow- } \\
\text { Induced } \\
\text { Vibrations }\end{array}$ & & & \\
\hline Volume number & & 96 & & 70 & 27 & 19 \\
\hline Page number & & $713-735$ & & $193-214$ & $838-847$ & $123-140$ \\
\hline Year & 2018 & 2008 & 1990 & 2017 & 2011 & 2001 \\
\hline Reference & [7] & [8] & [9] & [10] & [11] & [12] \\
\hline Authors & $\begin{array}{l}\text { N.B. Khan, Z. } \\
\text { Ibrahim, } \\
\text { L.T.T. } \\
\text { Nguyen, M.F. } \\
\text { Javed and M. } \\
\text { Jameel }\end{array}$ & $\begin{array}{l}\text { A. Mittal and } \\
\text { V. Kumar }\end{array}$ & $\begin{array}{c}\text { H. } \\
\text { Sayyaadi } \\
\text { and A. } \\
\text { Motekalle } \\
\text { m, }\end{array}$ & $\begin{array}{l}\text { M.C. Ong, T. } \\
\text { Utnes, L.E. } \\
\text { Holmedal, D. } \\
\text { Myrhaug, and } \\
\text { B. Pettersen, }\end{array}$ & $\begin{array}{l}\text { R.M. Stringer, J. } \\
\text { Zang and A.J. } \\
\text { Hillis, }\end{array}$ & $\begin{array}{c}\text { G.F. Roseri, } \\
\text { G. Vaz and } \\
\text { A.L.C. } \\
\text { Fujarra }\end{array}$ \\
\hline Journal title & PLoS One & $\begin{array}{l}\text { J FLUID } \\
\text { STRUCT }\end{array}$ & IJCOE & MAR STRUCT & Ocean Eng. & \\
\hline $\begin{array}{c}\text { Book title, } \\
\text { Proceedings title }\end{array}$ & & & & & & OMAE2012 \\
\hline Volume number & 12 & 5 & 4 & 22 & 87 & \\
\hline Page number & & $717-736$ & $1-10$ & $142-153$ & $1-9$ & \\
\hline Year & 2017 & 2001 & 2016 & 2009 & 2014 & \\
\hline
\end{tabular}

